\title{
Overview of Estimation Methods for Industrial Dynamic Systems
}

John Hedengren

Brigham Young University, john.hedengren@byu.edu

Ammon Eaton

Brigham Young University

Follow this and additional works at: https://scholarsarchive.byu.edu/facpub

Part of the Chemical Engineering Commons

\section{Original Publication Citation}

Hedengren, John D., and Ammon N. Eaton. "Overview of estimation methods for industrial dynamic systems." Optimization and Engineering (2015): 1-24.

\section{BYU ScholarsArchive Citation}

Hedengren, John and Eaton, Ammon, "Overview of Estimation Methods for Industrial Dynamic Systems" (2015). Faculty Publications. 1668.

https://scholarsarchive.byu.edu/facpub/1668

This Peer-Reviewed Article is brought to you for free and open access by BYU ScholarsArchive. It has been accepted for inclusion in Faculty Publications by an authorized administrator of BYU ScholarsArchive. For more information, please contact ellen_amatangelo@byu.edu. 


\title{
Overview of Estimation Methods for Industrial Dynamic Systems
}

\author{
Ammon N. Eaton · John D. Hedengren
}

Received: date / Accepted: date

\begin{abstract}
Measurement technology is advancing in the oil and gas industry. Factors such as wireless transmitters, reduced cost of measurement technology, and increased regulations that require active monitoring tend to increase the number of available measurements. There is a clear opportunity to distill the recent flood of measurements into relevant and actionable information. Common methods to do this include a filtered bias update, Implicit Dynamic Feedback, Kalman Filtering, and Moving Horizon Estimation. The purpose of these techniques is to validate measurements and align imperfect mathematical models to the actual process. Additionally, they can determine a best-estimate of the current state of the process and any potential disturbances. These methods allow potential improvements in earlier detection of disturbances, process equipment faults, and improved state estimates for optimization and control.
\end{abstract}

Keywords Dynamic data reconciliation $\cdot$ Wired drillpipe $\cdot$ Industrial data $\cdot$ Moving horizon estimation $\cdot$ Kalman filter

\author{
A.N. Eaton \\ Brigham Young University \\ $350 \mathrm{CB}$ \\ Provo, UT 84602 \\ Tel.: +1-801-680-1039 \\ Fax: +1-801-422-0151 \\ E-mail: ammon@byu.edu \\ J.D. Hedengren \\ Brigham Young University \\ $350 \mathrm{CB}$ \\ Provo, UT 84602 \\ Tel.: +1-801-477-7341 \\ Fax: +1-801-422-0151 \\ E-mail: john.hedengren@byu.edu
}




\section{Introduction}

Over the past 10 years many sectors of the oil and gas industry have seen a dramatic increase in the number and quality of available measurements. To capture the benefits of increased available measurements, the information must be distilled into relevant and actionable information [53,31]. This paper reviews the current industrial practice for estimation in the oil and gas refining, chemicals, exploration, and production sectors and provides guidance on model accuracy requirements for satisfactory control performance.

One opportunity is the increase in the available bandwidth to monitor the drilling process with along string and down-hole measurements to monitor pressure, vibration, temperature, and orientation. New technology has been deployed to drastically increase the data transmission rate to the Bottom Hole Assembly (BHA) or along the drill string. Mud pulsing was previously the most common form of communication in which 3-45 bits per second could be transmitted from the BHA to the surface monitoring system via a series of pressure waves through the inner pipe. In addition to providing a communication pathway, the pumped mud removes tailings and cools the drill bit. As the depth of drilling increases, the attenuation of mud pulses increases and mud pulse data is frequently unavailable. Recently, wire-in-pipe technology has increased this rate by approximately 10,000 times (see Figure 1) [28,35].

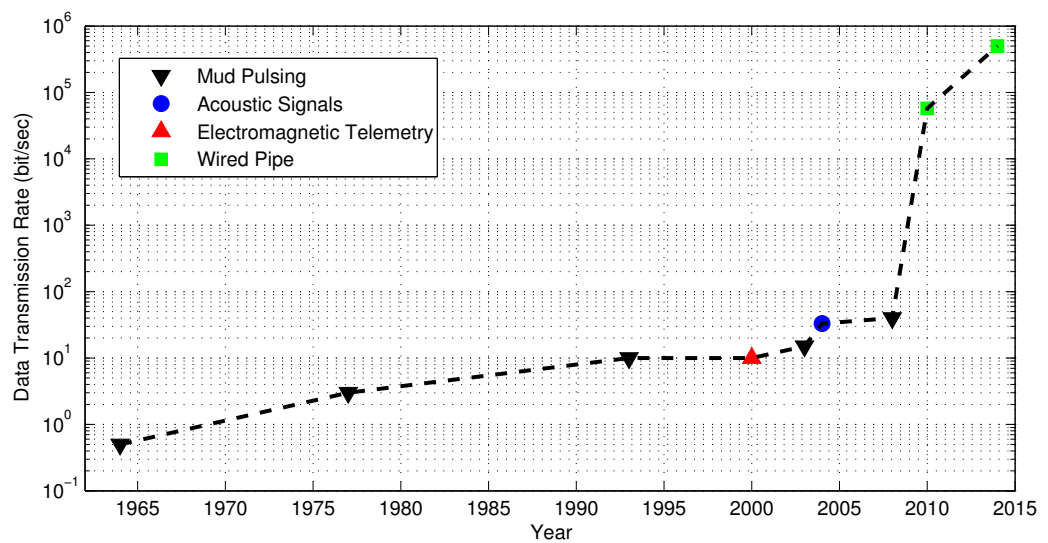

Fig. 1 Best available data transmission rates in drill strings [22,19]. The recent increase in throughput and bi-directional communication has created a new opportunity for better utilizing the information. Without interpretation, the increased data does not necessarily lead to increased understanding or value.

This increase in information allows two-way communication and presents opportunities for improved monitoring and control of directional, managed pressure, and under-balanced drilling $[48,49,36]$. Similar improvements in measurement technologies are occurring in other parts of the oil and gas industry. This paper reviews estimation techniques to garner the most useful data possible. These include a filtered 
bias update, Implicit Dynamic Feedback, Kalman Filtering, and Moving Horizon Estimation [3].

Moving Horizon Estimation (MHE) is an optimization approach that aligns process models with available measurements to determine a best estimate of the current state of the process and any potential disturbances. This presents the opportunity for earlier detection of disturbances such as gas influx into the bore-hole, process equipment faults, and improved state estimates for process automation. Explicit approaches commonly used in current practice, such as measured variable bias updating and Kalman filters, are compared to MHE approaches. Formerly, the downside to MHE approaches was the increased computational load required to solve the problem and the difficulty to obtain optimal tuning. This paper discusses techniques to overcome both of these obstacles to enable fast and reliable solutions that are tuned to optimally utilize measurement information in model predictive applications.

\subsection{Time-Scales of Process Monitoring}

Measurements of slow or fast processes pose unique challenges. The slow fouling of a heat exchanger [52] or the fast build-up of hydrates [15] are two examples of processes with different process time constants. With fouling or plugging as one of the top loss categories industry-wide, there are many opportunities for utilizing measurement technology to monitor the short or long term effects of these disturbances [23]. In particular, deep-sea pipeline monitoring poses a challenge due to the remote environment, intermittent weather incidents, and gradual fatigue factors [6]. There is a need for improved monitoring of existing and new projects to give insight into the conditions that lead to failure. Analytical models utilize the data to monitor and control the operational integrity for flow assurance and riser integrity [9].

\subsubsection{Frequency of Optimization Updates}

Before discussing techniques for measurements, it is informative to review the corresponding optimization applications. Optimization can occur after a model is synchronized to available process measurements or inputs. Process optimization is used in the oil and gas industry at various phases of the process lifecycle. As shown in Figure 2, optimization of process design occurs once at the beginning of the lifecycle [12]. This may include sizing of vessels, valves, etc. Optimization is also used to guide flow of products with Supply Chain Optimization. This may occur on a weekly to monthly basis $[46,37]$. Dynamic optimization is concerned with long time periods as well and covers processes such as defouling, turn-around operations, and production scheduling. On an hourly basis, Real-Time Optimization (RTO) with large-scale steady state models [24] is used to determine new targets for plant-wide operations [8]. On the second to minute time-scales, the steady-state conditions from the RTO application are passed to Model Predictive Control (MPC) applications that dynamically drive the process to new target values [55,20]. Recent work involves passing not only the new target values but also the economics from the RTO applications to the MPC applications as well $[43,10]$. Ensemble methods increase the reliability of the control 
methods, much like redundant sensors or physical equipment increase the reliability of operations by making the system less sensitive to a single failure [54].

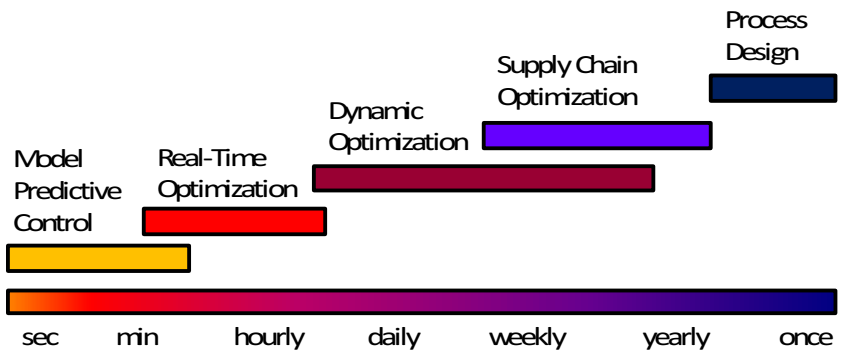

Fig. 2 Time-scales of optimization technologies applied in oil and gas industry.

\subsubsection{Frequency of Model and Measurement Alignment}

Just as optimization is applied at varying time-scales, measurement reconciliation is performed at varying time-scales that are analogous to the optimization approaches (see Figure 3).

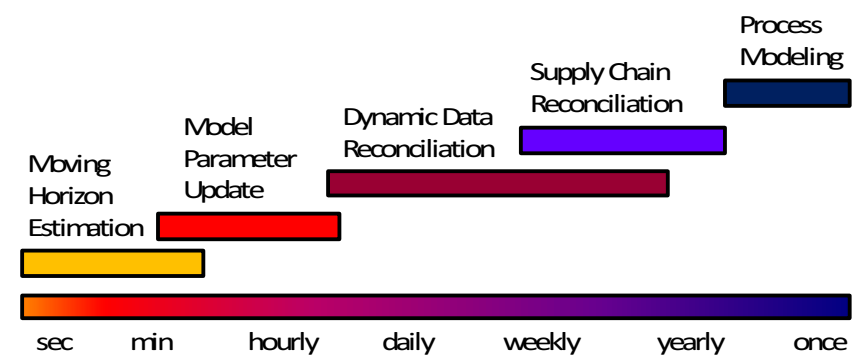

Fig. 3 Time-scales of measurement reconciliation applied in the oil and gas industry.

A sufficiently accurate model is required to optimize the design or control a process. During the lifecycle of a facility, modeling is typically conducted during the design and start-up of a new process. Data from other related processes are typically used to generate an initial process model which is then refined after the process unit comes online. Supply chain reconciliation seeks to align a model to the available inventories, capacities, and constraints [25]. Dynamic data reconciliation is used for large-scale dynamic models over long time horizons [27,29,1,56,38]. It is used in conjunction with dynamic optimization to align the model parameters with dynamic data [50]. For RTO applications, a precursor step is to adjust fouling factors, 
tray efficiencies, and other parameters with parameter estimation [8]. This parameter estimation may include single or multiple steady-state snapshots or the process measurements. One restriction is that the process must be at steady-state for the parameter estimation. Finally, MHE is a multi-variable approach for optimal measurement reconciliation in a dynamic model [42]. MHE applications are typically performed on a time-scale faster than that of the process time constant of interest. It typically executes in the range of seconds to minutes and can be used to provide state and parameter updates to MPC applications.

\subsection{Overview}

This paper is a review of strategies to incorporate measurements in optimization and monitoring applications. The mathematical models used in these applications have unmeasured or unmodeled disturbances that cause the model predictions to drift from actual values. This realignment of model and measurement can occur with a variety of techniques ranging from simplified to complex. When the application provides information in real-time, the results must be returned within a specified cycle time. Details on efficient implementation of the techniques are also presented in this paper with two motivating applications.

The focus of this paper is on measurement reconciliation for fast time processes in the range of seconds to minutes. New and established techniques are discussed that improve the information extraction from measurements to allow a fundamental understanding of a process.

\section{Numerical Solution with Dynamic Models}

The approach taken in this paper uses a simultaneous solution method as opposed to a sequential method to solve the model equations and objective function [45,47]. The general model form consists of nonlinear differential and algebraic equations (DAEs) in open equation format as shown in Equation 1.

$$
\begin{aligned}
& 0=f\left(\frac{\partial x}{\partial t}, x, u, d\right) \\
& 0=g(y, x, u, d) \\
& 0 \leq h(x, u, d)
\end{aligned}
$$

The optimizer calculates future states in the horizon that are uniquely specified by the initial state $x_{0}$, a given sequence of inputs $u=\left(u_{0}, u_{1}, \ldots, u_{n-1}\right)$, and a calculated set of disturbances $d=\left(d_{0}, d_{1}, \ldots, d_{n-1}\right)$. In Figure $4, u$ and $d$ are shown as discrete values over the horizon. Variables calculated from differential and algebraic equations are continuous over the time horizon. The solution of the open equation system is accomplished by converting the differential terms to algebraic equations with orthogonal collocation on finite elements [7], also known as direct transcription [11]. Order reduction may assist in understanding the most important states that dominate the system dynamics [16], but, the full system can typically be solved directly. 


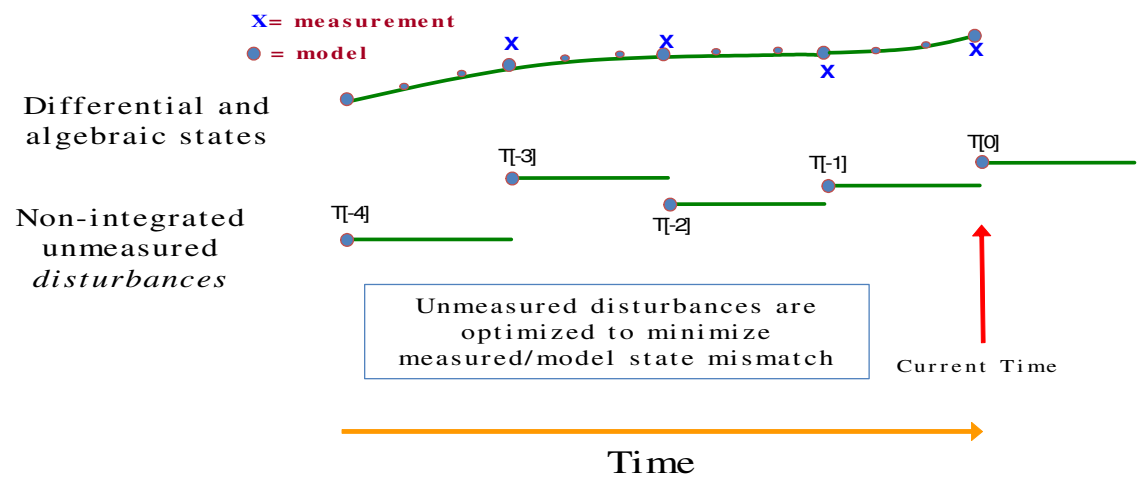

Fig. 4 Dynamic equations are discretized over a time horizon and solved simultaneously.

The solution of the estimation problem is solved with an implicit solution technique such as large-scale NLP solvers [27,2]. Other methods include the direct shooting approaches [21] or the explicit solution $[40,17]$ for simplified problems. The difference between competing implicit solution techniques is how the state equations are satisfied. Direct single or multiple shooting solves the state equations to a convergence tolerance for every iteration. Using orthogonal collocation on finite elements, the state equations are only satisfied at a converged solution. This generally leads to a more efficient solution, especially for large-scale problems with many decision variables [14].

\section{Review of Current Strategies}

Advanced Process Control (APC) has produced significant benefits in the oil and gas industry, especially in refining and chemicals and more limited in exploration and production $[39,58]$. Simpler control applications such as PID controllers are often preferred in most single-input, single-output controllers. Measurement reconciliation also ranges from simple to complex [51]. Simple techniques include filtered bias updates or Implicit Dynamic Feedback (IDF). More complex strategies include Kalman filtering and MHE. Each of these techniques are discussed below.

\subsection{Filtered Bias Update}

A predominant approach for measurement feedback into many of the popular APC commercial packages continues to be a filtered bias update [39]. Adding an output constant or integrating disturbance introduces insignificant computational overhead and is easy to tune. In the case of a constant disturbance, an additive model bias $b$ is updated at iteration $n$ with a filter $\alpha$ as shown in Equation 2

$$
b_{n}=\alpha\left(z_{n}-y_{n}\right)+(1-\alpha) b_{n-1}, \quad 0 \leq \alpha \leq 1
$$


Table 1 Filtered Bias Update Trade-offs

\begin{tabular}{l}
\hline Strengths of the Filtered Bias Update \\
\hline Incorporated with many popular APC commercial packages \\
Single tuning parameter, $\alpha$, that balances noise rejection with measurement tracking speed \\
Insignificant computational overhead \\
\hline Drawbacks of the Filtered Bias Update \\
\hline No capability to estimate parameters or unmeasured disturbances \\
No consideration of multivariable effects \\
Offset is present for integrating disturbances \\
Physical constraints may be violated
\end{tabular}

In this case, the difference between the measured state $z_{n}$ and the predicted model $y_{n}$ is used to update the offset of a controlled variable initial condition. With a weak filter with $\alpha$ near 1, almost all of the measurement value is accepted for updating the model predicted value. Strong filters that accept less of the measured value may cause the corresponding APC application to respond slowly to unmodeled disturbances. The value of $\alpha$ is typically chosen to balance noise rejection with speed of reaction. The strengths and drawbacks of the filtered bias update are summarized in Table 1.

In order for the bias to be updated, certain qualifications may also be set to detect bad measurements. These qualifications are commonly upper and lower validity limits as well as a rate of change validity limit. The validity limits are applied to either the raw measurement or the raw bias. If any of the validity limits are violated, the measurement is rejected and the bias value remains constant. Rate of change validity limits are frequently set too restrictively for process upset conditions such as shutdown or startup, necessitating the need for operator intervention or automatic application switching to manual control.

\subsection{Implicit Dynamic Feedback}

Implicit Dynamic Feedback (IDF) estimates unmeasured disturbances related to the predictions of the measured state variables. IDFpairs a single measurement with a single unmeasured disturbance variable. The analogy to control is the Single Input, Single Output (SISO) controllers such as the ubiquitous PID controller. In the case of IDF, the unmeasured disturbance variable is adjusted to align the model with a process measurement. IDFconsists of two equations that can be solved simultaneously with the control problem over a preceding horizon interval. The IDFequations are similar to a proportional integral (PI) controller. The IDF input is the difference between the measured state $z$ and model state $y$. This is similar to the PI controller with a setpoint $(S P=z)$ and process variable $(P V=y)$. The output is an unmeasured disturbance variable $d$ of the model and is analogous to the PI controller as the manipulated variable. This disturbance variable is adjusted proportionally to the current and integrated measurement errors, as shown in Equation 3a. However, Equation 3a is not implemented in practice because of the integral term. To overcome this, the 


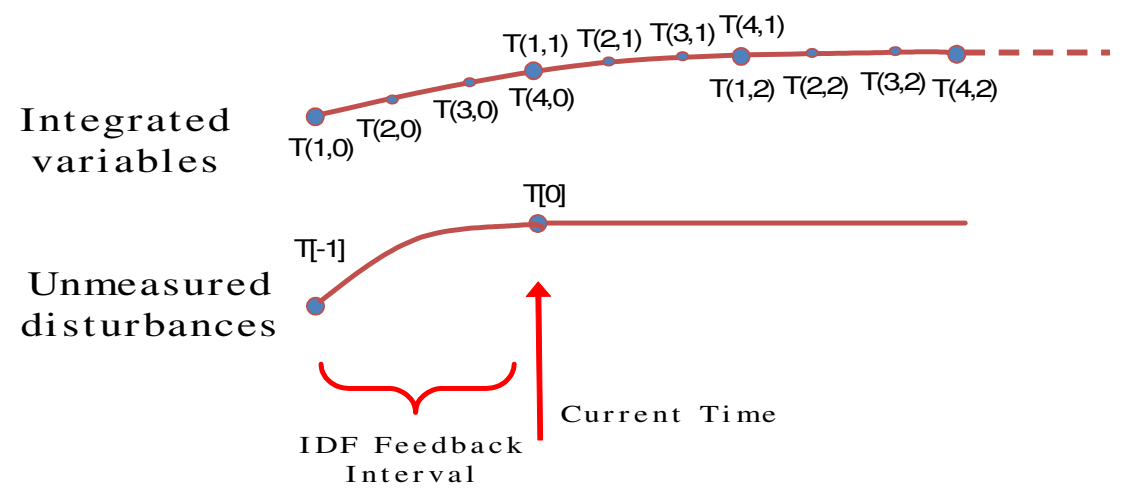

Fig. 5 IDFhorizon with simultaneous estimation and dynamic optimization.

Table 2 Implicit Dynamic Feedback Trade-offs

Strengths of IDF

Only two differential equations are required to implement IDF

Similar tuning to a PID controller

Two intuitive parameters trade-off speed versus stability

Drawbacks of IDF

Restricted to one-to-one pairing of a measurement to an unmeasured disturbance

Potential wind-up of the integral term

One step estimation horizon gives parameter values that may not project into the future for predictive applications (e.g. MPC)

Physical constraints cannot be enforced

integral term ' $I$ ' is differentiated and the IDFequations are solved as two separate expressions (see Equation 3b).

$$
\begin{gathered}
d=K_{c}(z-y)+\frac{K_{c}}{\tau_{I}} \int_{t=0}^{T}(z-y) d t \\
d=K_{c}(z-y)+\frac{K_{c}}{\tau_{I}} I, \quad \frac{\partial I}{\partial t}=(z-y)
\end{gathered}
$$

The tuning parameters for IDFare $K_{c}$ and $\tau_{I}$, the same as a PI controller. Using a large value of $\tau_{I}$ and small $K_{c}$ has the effect of heavily filtering the error term for feedback. In this case the algorithm will take longer to match the plant. Using these tuning parameters and knowing the quality and types of measurements enables trading off of speed of tracking the process versus stability concerns. The advantages and disadvantages of IDFare listed in Table 2.

IDFhas been successfully used for many years to provide on-line estimation measurement biases, catalyst activities, kinetic parameter adjustment factors and heat transfer coefficients. However, IDFis limited to a past horizon length of one, pairing of only one measurement to one disturbance, and the inability to handle constraints. 


\subsection{Kalman Filter}

With a Kalman filter, sequential measurements are used to obtain the state of the system with a linear model. To obtain this model, Jacobian information from Equation 1 are rearranged into the discrete state space form (see Equation 4) where $A, B, C$ are constant matrices, $u$ is the manipulated variable vector, $x$ is the state vector, $y$ is the vector of model outputs. In this case, the subscript $n$ refers to the time step at which the model is computed.

$$
\begin{gathered}
x_{n+1}=A x_{n}+B u_{n} \\
y_{n}=C x_{n}
\end{gathered}
$$

The horizon of measurements is combined mathematically to generate the system's state at the current time with the Kalman filter as shown in Equation 5. The Kalman filter is divided into 4 subsets of equations. In Equation 5a the states $\bar{x}$ and covariance $\bar{P}$ are predicted in the absence of new measurement information. In the next step (see Equation $5 b$ ), the predictions are compared to the measured values. The innovation $\tilde{\delta}$ and innovation covariance $S$ are the comparison of the model predictions to the measured reality. The innovation covariance $S$ and covariance prediction $\bar{P}$ are then used to calculate the Kalman gain $K$ in Equation 5c. As a final step, the new state and covariance estimates are computed in Equation 5d. The Kalman gain relates the fraction of the innovation $\tilde{\delta}$ and state prediction $\bar{x}$ that are used to construct the new state estimate $x_{n}$. Similarly, the Kalman gain relates the predicted covariance prediction to the new covariance prediction. Note that the covariance update is independent of the measurement values $z_{n}$ and the time evolution is only a function of constant matrices.

$$
\begin{gathered}
\bar{x}=A x_{n-1}+B u_{n} \\
\bar{P}=A P_{n-1} A^{T}+Q \\
\tilde{\delta}=z_{n}-C \bar{x} \\
S=C \bar{P} C^{T}+R \\
K=\bar{P} C^{T} S^{-1} \\
x_{n}=\bar{x}+K \tilde{\delta} \\
P_{n}=(I-K C) \bar{P}
\end{gathered}
$$

The Kalman filter is optimal for unconstrained, linear systems subject to known normally distributed state and measurement noise [13]. The Extended Kalman Filter (EKF) or Unscented Kalman Filter (UKF) are an attempt to extend these techniques to nonlinear systems. A summary of the trade-offs related to the Kalman filter are listed in Table 3.

EKF is able to predict the nonlinear state evolution by re-linearizing the model at each time instant. Some effort has been made to incorporate constraints with EKF 
Table 3 Kalman Filter Trade-offs

\begin{tabular}{l}
\hline Strengths of Kalman Filtering \\
\hline Optimal estimator for linear systems without constraints \\
Solution approach is accomplished through matrix multiplications \\
Covariance estimate provides confidence interval for state estimate \\
\hline Drawbacks of Kalman Filtering \\
\hline Restricted to linearized model state updates \\
Physical constraints cannot be enforced \\
Can only estimate model states, not model parameters \\
Cannot utilize infrequent measurements or those with variable time delay \\
Occasional bad data may result in inaccurate state estimates \\
\hline
\end{tabular}

although the state augmentation strategy for parameter estimation is still a limitation [57]. Kalman based techniques suffer from a number of limitations. For nonlinear or constrained systems, optimization techniques such as MHE are better suited to providing an estimate of the true system state.

\subsection{Squared Error MHE}

MHE outperforms the Extended Kalman Filter (EKF) in the presence of constraints [13]. Recent advances in computational capability and methods have improved the application of MHE to fast [26] and large-scale industrial systems [41]. Just as APC has demonstrated significant benefits by considering multivariate relationships [4], MHE is better able to utilize measurements and deliver a more accurate description of the current state of the process and disturbances [44].

By using an optimization framework, the model and measurement values are aligned and present detailed information about the system dynamics. This optimization framework uses a receding horizon of process measurements. MHE attempts to optimally estimate the true state of the dynamic system, given a real-time stream of measurements and a model of the physical process. Offset free estimation and control is achieved by adding as many disturbance variables as the number of measurements $[30,34,33]$. The MHE objective function is posed as a squared error minimization to reconcile the model with measured values. The trade-offs for MHE with a squared objective function are summarized in Table 4.

In a MHE form amenable to real-time solution, the unmeasured disturbance variables $d$ are adjusted to match the continuous model to discrete measured values [41].

$$
\begin{aligned}
\min _{d} \Phi & =\left\|\frac{z-y}{y}\right\|_{Q_{y}}^{2}+\|d-\hat{d}\|_{Q_{d}}^{2} \\
\text { s.t. } \quad 0 & =f(\dot{x}, x, u, d) \\
& =g(z, x, u, d) \\
0 & \leq h(x, u, d)
\end{aligned}
$$

In Equation 6, $\Phi$ is the objective function value, $z$ is a vector of measurements at all nodes in the horizon $\left(z_{0}, \ldots, z_{n}\right)^{T}, y$ is a vector of model values at the sampling times $\left(y_{0}, \ldots, y_{n}\right)^{T}, Q_{y}$ is the inverse of the measurement error covariance, $f$ is a vector 
Table 4 Trade-offs for MHE with a Squared Error Objective

\begin{tabular}{l}
\hline Strengths of MHE (Squared Error) \\
\hline Least squares is an intuitive objective that is simple to implement \\
Model constraints can be added to model to improve the estimation accuracy \\
Optimal tuning has been established [32] \\
\hline Drawbacks of MHE (Squared Error) \\
Poor rejection of outliers or infrequent bad values common with real data \\
Difficult to obtain good estimates of $P_{0}, Q$, and $R$ \\
Dense tuning matrices impractical for large-scale systems \\
Iterative optimization solution that may fail to converge in the required cycle time \\
\hline
\end{tabular}

of model equation residuals, $x$ represents the model states, $u$ is the vector of model inputs, $d$ is the vector of model parameters or unmeasured disturbances, $\hat{d}$ is the vector of prior unmeasured disturbances, $Q_{d}$ is a matrix for the weight on changes of disturbance variables, $g$ is an output function, and $h$ is an inequality constraint function.

A graphical representation of the MHE squared error reconciliation is shown in Figure 6. The objective for this measured value is a quadratic function with the minimum target between the previous model and measured values.

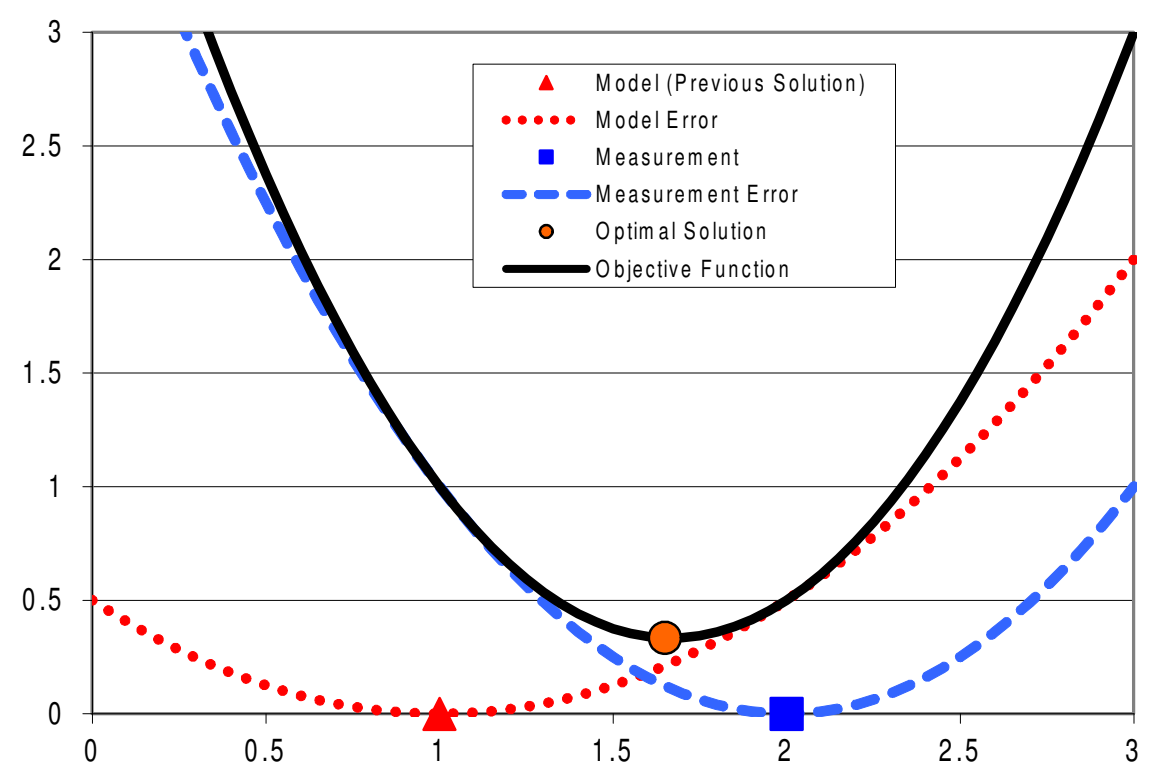

Fig. 6 Graphical representation of the squared error for a single measurement in the horizon. 
The full estimation problem allows violation of the state constraints [44]. State equality constraints are relaxed and violations are penalized in the objective function. Without $d$ the optimization problem found in Equation 6 does not allow state transition error because the state equations are exactly satisfied at a converged solution [5]. This can be overcome by creating a discontinuous state $y$ and disturbance $d$ with an additional equation $y=x+d$ for each state subject to state noise. This allows discontinuities in the $y$ states while preserving the continuity of the $x$ states. However, allowing state noise is undesirable when employing first principles models. For material and energy balances, allowing state noise reduces the predictive potential of the model. Instead, the only decision variables are selected as $x_{0}$ and $d$ instead of $\left(x_{0}, \ldots, x_{n}, p\right)$ as in the full MHE problem.

As the estimation horizon increases, the sensitivity of the solution at $x_{n}$ to $x_{0}$ decreases. With a first-order approximation, the value of the final state $x_{n}$ sensitivity decreases by $e^{-\frac{t}{\tau}}$ where $\tau$ is the approximate process time constant. For sufficiently long time horizons, it is then only $d$ that has a significant effect on the current model state. Thus, it is generally unnecessary to estimate the initial states $x_{0}$ as degrees of freedom in the optimization problem.

\section{$3.5 \ell_{1}$-Norm MHE}

A new form of MHE has been used in industry for a number of years that overcomes some of the limitations of the squared error MHE approach [18]. The objective function in Equation 7 is implemented in a form that is amenable to numerical solutions of large-scale models. The use of an absolute value function is avoided by instead solving inequality constraints with slack variables. The slack variables and inequalities create an objective function that is smooth and continuously differentiable as a requirement for large-scale Nonlinear Programming (NLP) solvers.

$$
\begin{array}{ll}
\min _{d} \Phi & =w_{m}^{T}\left(e_{U}+e_{L}\right)+w_{p}^{T}\left(c_{U}+c_{L}\right) \\
\text { s.t. } & 0=f(\dot{x}, x, u, p, d) \\
& 0=g(y, x, u, d) \\
& 0 \leq h(x, u, d) \\
& \\
& e_{U} \geq y-y_{U} \\
& e_{L} \geq y_{L}-y \\
& c_{U} \geq y-\hat{y} \\
& c_{L} \geq \hat{y}-y \\
& e_{U}, e_{L}, c_{U}, c_{L} \geq 0
\end{array}
$$

Here, $\Phi$ is the objective function value, $z$ is a vector of measurements at all nodes in the horizon $\left(z_{0}, \ldots, z_{n}\right)^{T}, y$ is a vector of model values at the sampling times $\left(y_{0}, \ldots, y_{n}\right)^{T}$, $\hat{y}$ is a vector of previous model values at the sampling times $\left(\hat{y}_{0}, \ldots, \hat{y}_{n}\right)^{T}, w_{m}$ is a vector of weights on the model values outside a measurement dead-band, $w_{p}$ is a vector of weights to penalize deviation from the prior solution, $f$ is a vector of model equation residuals, $x$ represents the model states, $u$ is the vector of model inputs, $d$ is the 
vector of model parameters or unmeasured disturbances, $g$ is an output function, $h$ is an inequality constraint function, $e_{U}$ and $e_{L}$ are slack variables to penalize model values above and below the measurement dead-band, and $c_{U}$ and $c_{L}$ are slack variables to penalize model value changes above and below the previous values.

A graphical representation of the MHE $\ell_{1}$-norm reconciliation is shown in Figure 7. Parameters are only adjusted if the measured value is more than the half of the dead-band away from the previous model value. Otherwise, the model is not adjusted because the measurement lies within the region of a flat objective function. In the case of Figure 7, the optimal solution lies at the edge of the measurement dead-band. This will always be the case for measurements that are more than half the dead-band distance from the prior model value.

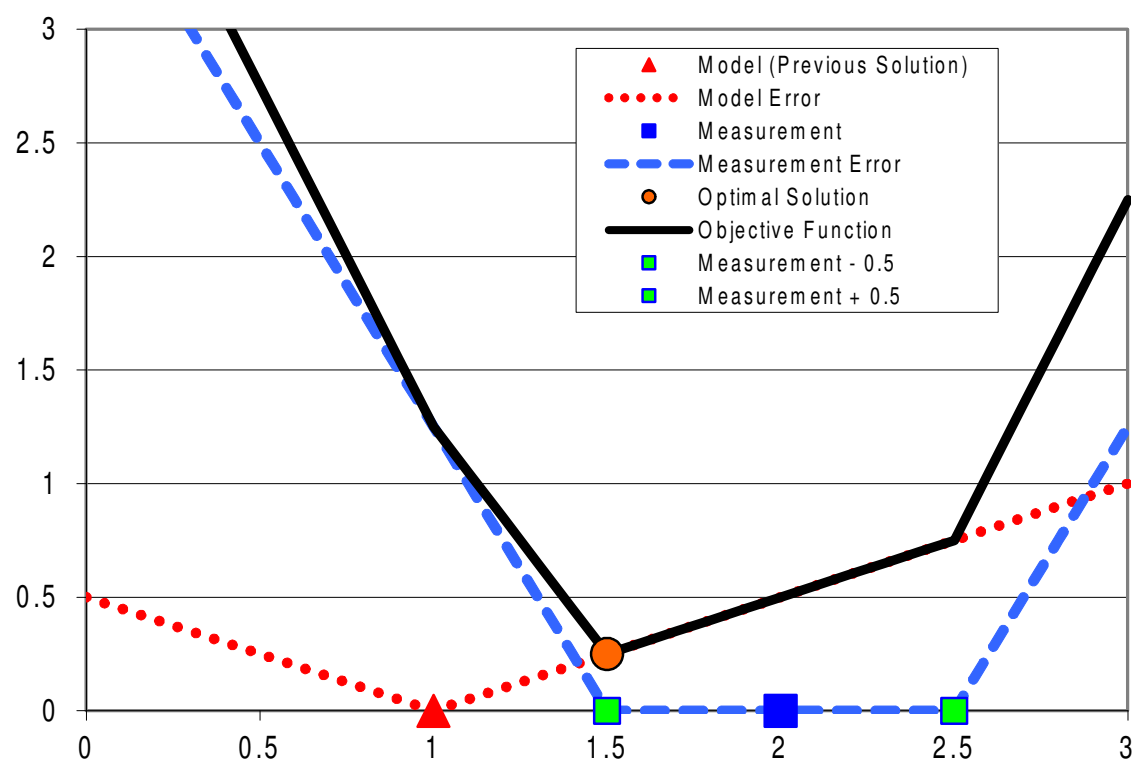

Fig. 7 Graphical representation of the MHE $\ell_{1}$-norm for a single measurement in the horizon.

The MHE $\ell_{1}$-norm objective has a number of advantages and challenges compared with other methods such as the Kalman filter or the MHE squared error. The next section details the trade-offs between the different techniques.

\subsubsection{MHE $\ell_{1}$-Norm Advantages}

An important MHE $\ell_{1}$-norm advantage is less sensitivity to data outliers, noise, and measurement drift [18]. This is important when dealing with industrial data where 
Table 5 Trade-offs for MHE with an $\ell_{1}$-norm Objective

\begin{tabular}{l}
\hline Strengths of MHE $\left(\ell_{1}\right.$-norm) \\
\hline Low sensitivity to data occasional bad data \\
Linear objective function and sparse tuning techniques improve scaling to large-scale systems \\
Explicit measurement dead-band for improved noise rejection \\
\hline Drawbacks of MHE $\left(\ell_{1}\right.$-norm $)$ \\
\hline Additional equality and inequality constraints and variables, leading to increased computation time \\
No optimal theory on best tuning parameters \\
Requires an iterative solver to reliably converge in a specified cycle time
\end{tabular}

instruments drift or fail. Gross-error detection can eliminate a majority of bad data. With MHE $\ell_{1}$-norm, any data that isn't filtered by gross-error detection has less impact on the parameter estimation and allows improved reliability of the solution. A squared error objective is more sensitive and disproportionately weights values that are far from the model predictions.

An additional advantage of the MHE $\ell_{1}$-norm is that only linear equations are added to the objective function. Without additional nonlinear expressions, the solution is generally easier for numerical solvers to find an optimal solution. In summary, the MHE $\ell_{1}$-norm optimization problem with measurement noise dead-band has a number of advantages over the MHE squared error form of the objective function. The trade-offs for MHE with an $\ell_{1}$-norm objective function are summarized in Table 5 .

\subsubsection{MHE $\ell_{1}$-Norm Challenges}

The challenges with the MHE $\ell_{1}$-norm optimization problem include increased complexity and size. Although the MHE $\ell_{1}$-norm uses only linear expressions in formulating an objective function, there are additional slack variables and inequality expressions, which increases the size of the optimization problem.

Many of the MHE $\ell_{1}$-norm challenges are due to the increased complexity in the solution techniques. Commercial and academic software has been developed to meet this challenge. The software used to generate the results in this paper is the APMonitor Modeling Language [18]. Filtered bias updating, Kalman filtering, IDF, and MHE are implemented in this web-services platform through MATLAB or Python.

\section{Managed Pressure Drilling Flow Estimation}

As an example application, consider the problem of determining the flow of mud through the return annulus of a drilling pipe. In the return line, there is typically a flow paddle that rotates proportional to the flow rate. This flow paddle measurement is not very accurate so additional information such as pit tank level can be used to infer the return flow. Additionally, in Managed Pressure Drilling (MPD), a choke valve is adjusted to maintain well pressure as shown in Figure 8. 


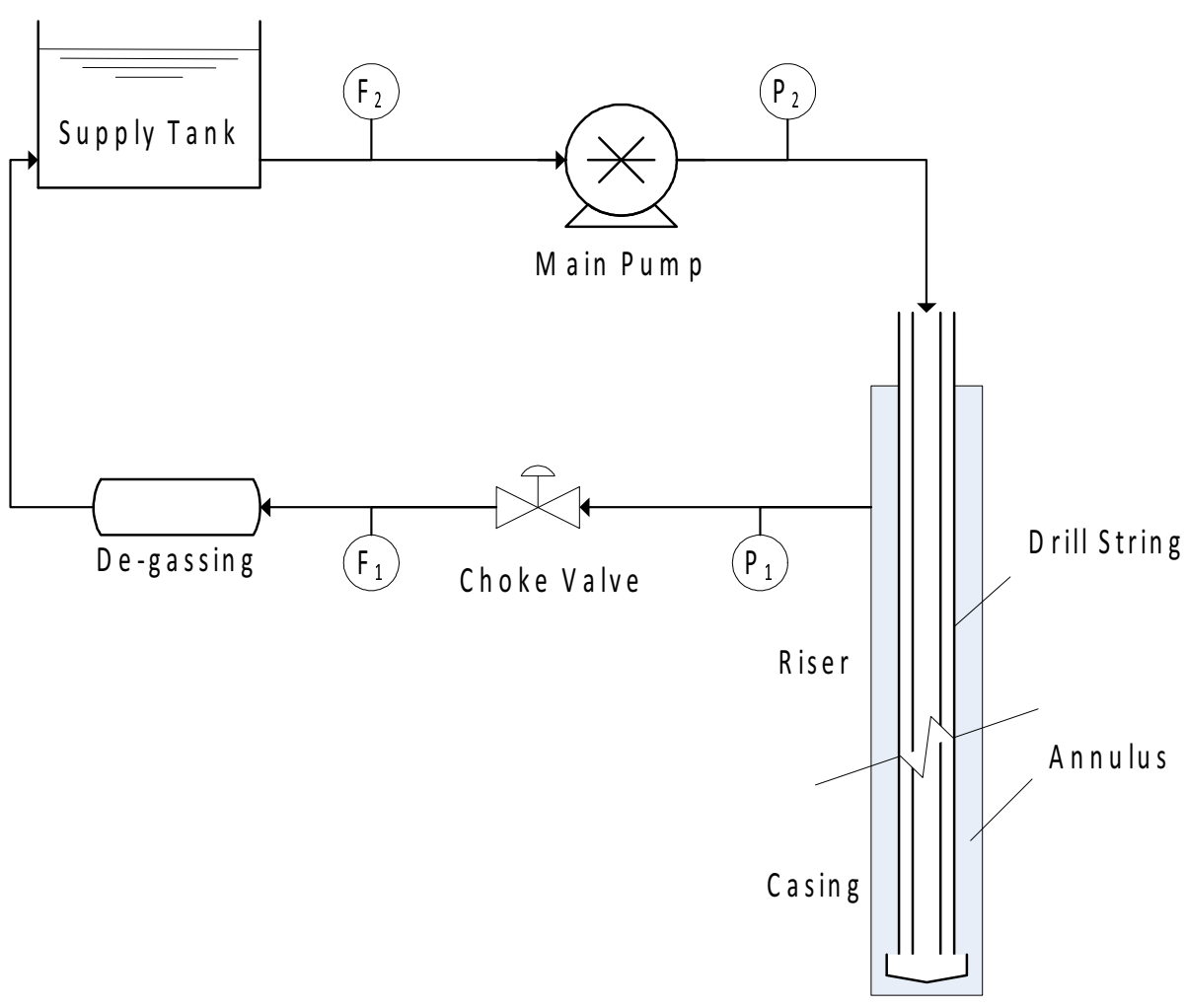

Fig. 8 Schematic of Managed Pressure Drilling.

The flow, pressure, and level measurements have noise, creating random fluctuations around the true values. The flow through the choke valve can also be estimated from the valve position and differential pressure across the valve (see Equation 8).

$$
\tau \frac{\partial q}{\partial t}+q=C_{v} f(l) \sqrt{\left(\frac{\Delta P_{v}}{g_{s}}\right)}
$$

For this example, the installed characteristic of the choke valve is assumed to be linear $(f(l)=l)$ and the valve is fast acting $(\tau=1 \mathrm{sec})$. Both the state and measurement noise are normally distributed with mean values of zero (see Figure 9). State noise has a standard deviation $\sigma_{q}=0.1$ and measurement noise has a standard deviation $\sigma_{r}=1.0$.

The Kalman filter updates the state estimates by operating in two phases: predict and update. In the prediction phase, the calculated flow is modified according to the equation that relates flow $q$ to the lift function $f(l)$ and the differential pressure, $\Delta P_{v}$. For Kalman filters, the equation must first be linearized. With Extended Kalman filters, the nonlinear equations are re-linearized about the current state estimate. The other parameters, including $\tau, C_{v}$, and $g_{s}$, are constants for a particular valve and 

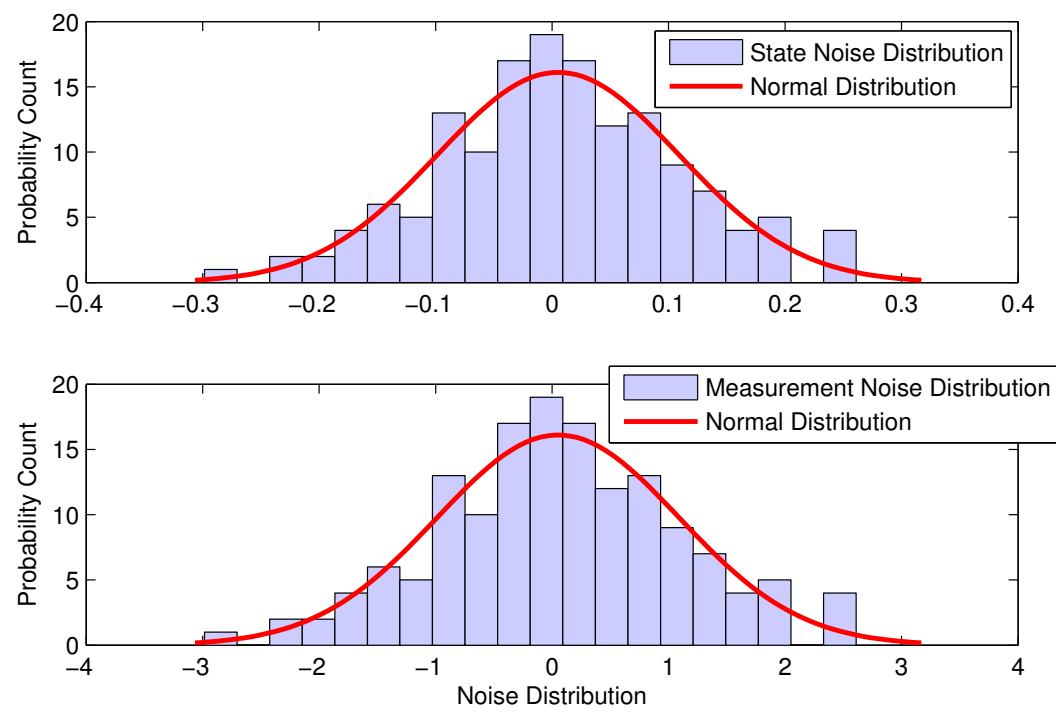

Fig. 9 Noise distributions of state and measurement noise. These distributions are used to optimally tune the estimators.

fluid. For systems with multiple measurements, the covariance is used to tune the Kalman filter. In this case with one measurement, the variance is used instead. This information is essential for optimizing the update phase; yet state and measurement covariance information can be difficult to obtain. The results of the Kalman filter with the upper and lower 95\% confidence intervals are shown in Figure 10.

In the update phase, a measurement of the flow is taken from the transmitter. Because of the noise, this measurement has some uncertainty. The calculated variance from the predict phase determines how much the new measurement affects the updated prediction. If the model prediction drifts away from the real flow, the measurements from the flow transmitter should pull the flow estimate back towards the real flow but not disturb it to the point of introducing all of the noise from the measurement.

This model update could also employ other measurements such as mud pump speed, choke pressure, or supply tank level to infer the flow rate across the valve. For this simple example, only the valve position and flow measurements are used to predict the flow with a linear, first-order correlation. Each of the five techniques discussed in this paper are compared over the same data set as shown in Figure 11.

The filtered bias update and IDFhave been tuned to give equivalent responses. After an initialization period, they also align exactly with the Kalman filter results because the Kalman gain becomes constant after the estimate of the covariance matrix $P_{n}$ also converges to a constant value. The first four methods including filtered bias update, IDF, the Kalman filter, and the squared error MHE (with one horizon step) can be tuned to give equivalent results for this single measurement case. Table 6 shows 


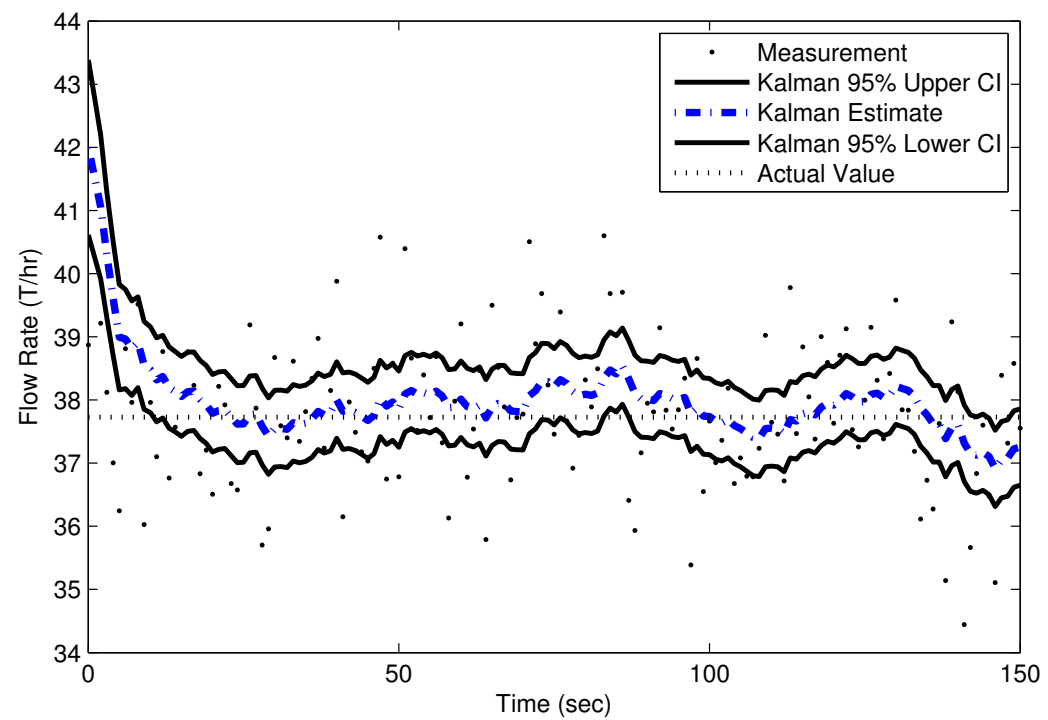

Fig. 10 The Kalman filter uses two phases, predict and update, to obtain an estimate of the true flow. During the predict phase, the model calculates an updated flow due to the latest reported model inputs. During the update phase, part of the flow measurement is used to update the state, inversely proportional to the variance of the measurement error.

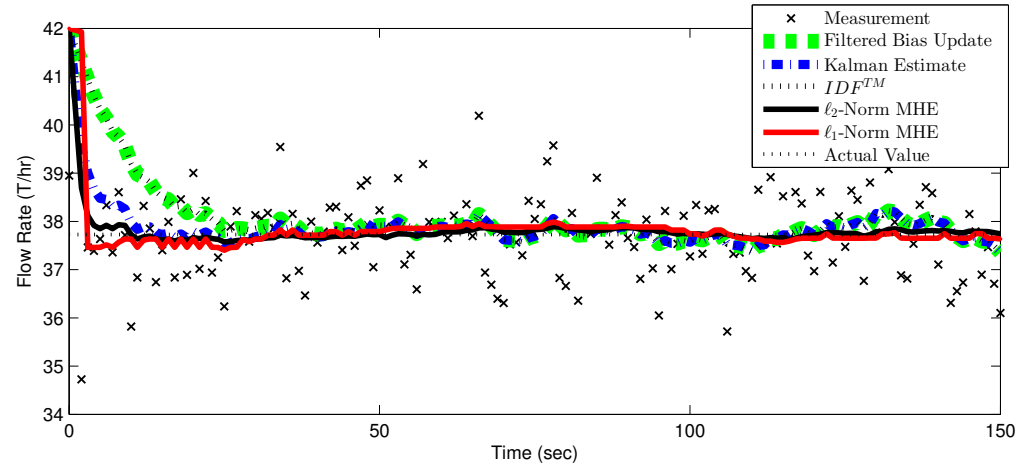

Fig. 11 Actual, measured, and estimated flows for filtered bias update, IDF, the Kalman filter, squared error MHE, and $\ell_{1}$-norm MHE.

the tuning values that make each of the estimators equivalent for this example case and in general.

In addition to signal loss, real data often contains bad data such as outliers, drift, and noise. Outliers do not typically fit a standard normal distribution but are instead drastic deviations from normal variation in the data. Outlier detection and removal is typically accomplished by setting rate of change limits, upper validity limits, and 
Table 6 Estimator Configuration Values

\begin{tabular}{lll}
\hline Estimation Method & Example Tuning & Equivalent Tuning for One Measurement \\
\hline Filtered Bias Update & $\alpha=0.0951$ & Set $\alpha$ equal to the Kalman Gain $K$ \\
IDF & $K_{c}=0.0951 e-10, \tau_{I}=1 e-10$ & Set $\frac{K_{c}}{\tau_{I}}$ equal to the Kalman Gain as $K_{c} \rightarrow 0$ \\
Kalman Filter & $P_{0}=0.5, Q=0.01, R=1.0$ & $\begin{array}{l}\text { Set } P_{0}=P_{\infty} \text { for equivalency to other methods } \\
\text { during initialization }\end{array}$ \\
Squared Error MHE & Horizon $=50, Q_{y}=100, Q_{d}=10$ & $\begin{array}{l}\text { For linear systems with quadratic objective } \\
\text { MHE reduces to Kalman Filter [42] }\end{array}$ \\
& Horizon $=50$ & $\begin{array}{l}\text { The } \ell_{1} \text {-norm MHE does not have equivalent } \\
\text { tuning correlations to the other methods }\end{array}$ \\
\hline
\end{tabular}

lower validity limits. This gross error detection eliminates many, but not all, of the data outliers. The effect of data outliers is shown in Figure 12 with the introduction of an outlier at cycle 50, drift starting at cycle 100, and increased noise starting at cycle 150 .

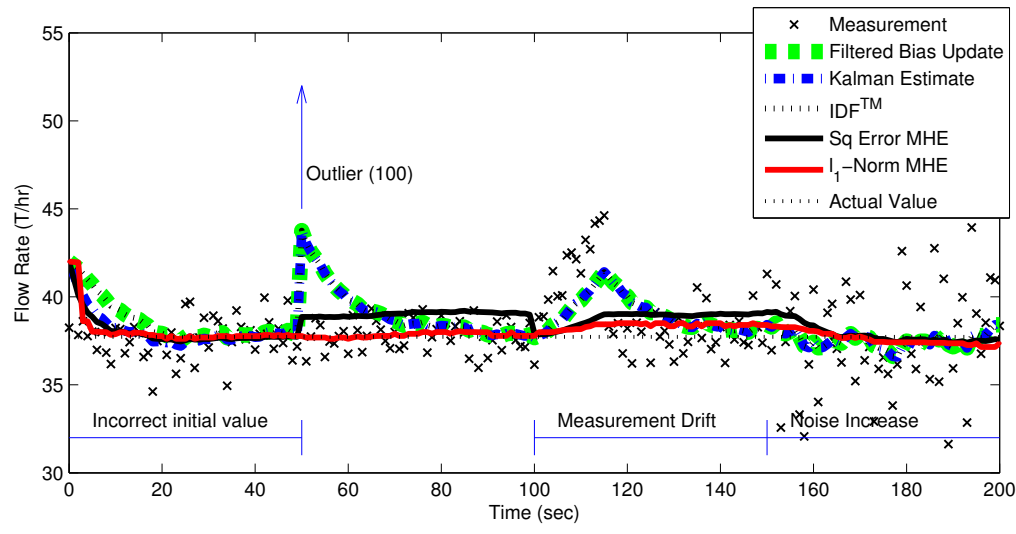

Fig. 12 Outlier effect on the filtered bias update, IDF, the Kalman filter, squared error MHE, and $\ell_{1}$-norm MHE. The $\ell_{1}$-norm MHE is least sensitive to brief periods of bad data.

The results with bad data with an outlier, drift, and noise clearly indicate that all state estimates, except the $\ell_{1}$-norm MHE, are significantly affected by the bad data points. The insensitivity to bad data is a key advantage of the $\ell_{1}$-norm MHE approach.

\section{Estimation for Control Relevant Models}

One important function of estimation methods is to improve the predictive qualities of a model prior to forward prediction methods such as MPC. Process gains and time constants are often used to characterize the relationship between a manipulated variable (MV) and associated controlled variable (CV) in control applications. An issue that occasionally arises in linear MPC applications is that the process conditions 
change and the model is no longer sufficiently predictive for satisfactory control performance. A simplified MPC application is developed in this section to test the limits of process/model mismatch in quantifying controller performance with varying combinations of process gains and dynamics.

In this MPC application, a first order linear process $(\tau d x / d t=-x+K u)$ with a gain $(K)$ of 1.0 and process time constant $(\tau)$ of $1.0 \mathrm{sec}$ is controlled. The control horizon is set to $4.0 \mathrm{sec}$ with a time discretization of $0.5 \mathrm{sec}$. The MPC controller minimizes the deviation of the $\mathrm{CV}$ from a target value of 5.0, starting from an initial condition of 0.0. The model gain and time constant are changed to incorrect values and the MPC performance is simulated over 20 control cycles. With a 0.5 sec cycle time, there is a total of $10 \mathrm{sec}$ simulated control time. The absolute value of the deviation from the target set point (5.0) is recorded for each combination of $K$ and $\tau$ values with a range of mismatch of 0.2 to 5.0 for each. Figures 13 and 14 show the control performance over the range of mismatched models applied in the MPC controller.

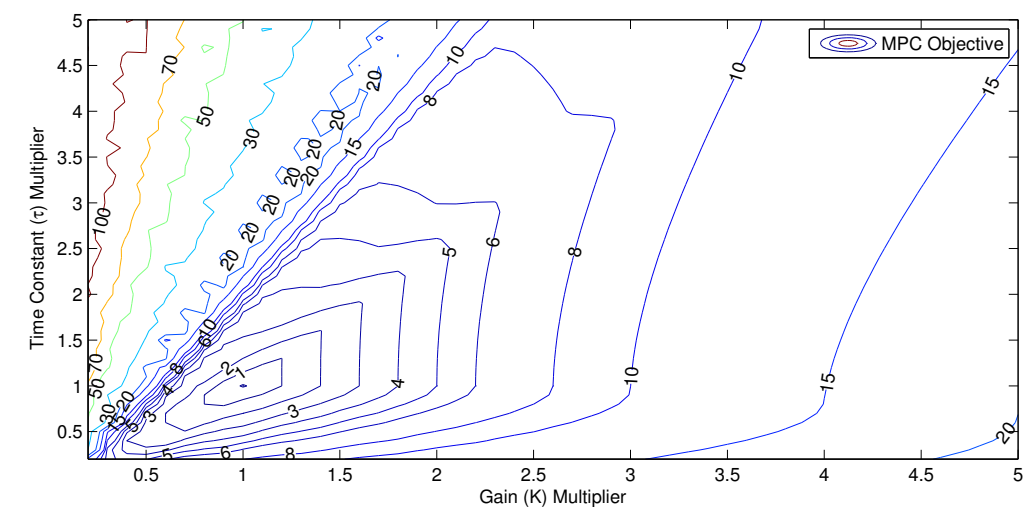

Fig. 13 Contour plot of the control objective with varying mismatch of the process gain and time constant.

If the model is used in a model predictive controller, a model gain that is lower than the actual process gain may cause controller oscillation and instability. A model gain that is higher than the actual process may cause sluggish control. Likewise, a model response (time constant) that is slower (higher time constant) than the actual process time constant tends to cause controller instability. On the other hand, a model response (time constant) that is faster (lower time constant) than the actual process tends to cause sluggish controller response. The combination of a low gain and high time constant leads to the highest objective function (poor performance and instability). On the other hand, a high gain and low time constant lead to sluggish control, but the controller is generally able to asymptotically drive the process to the correct set point. 


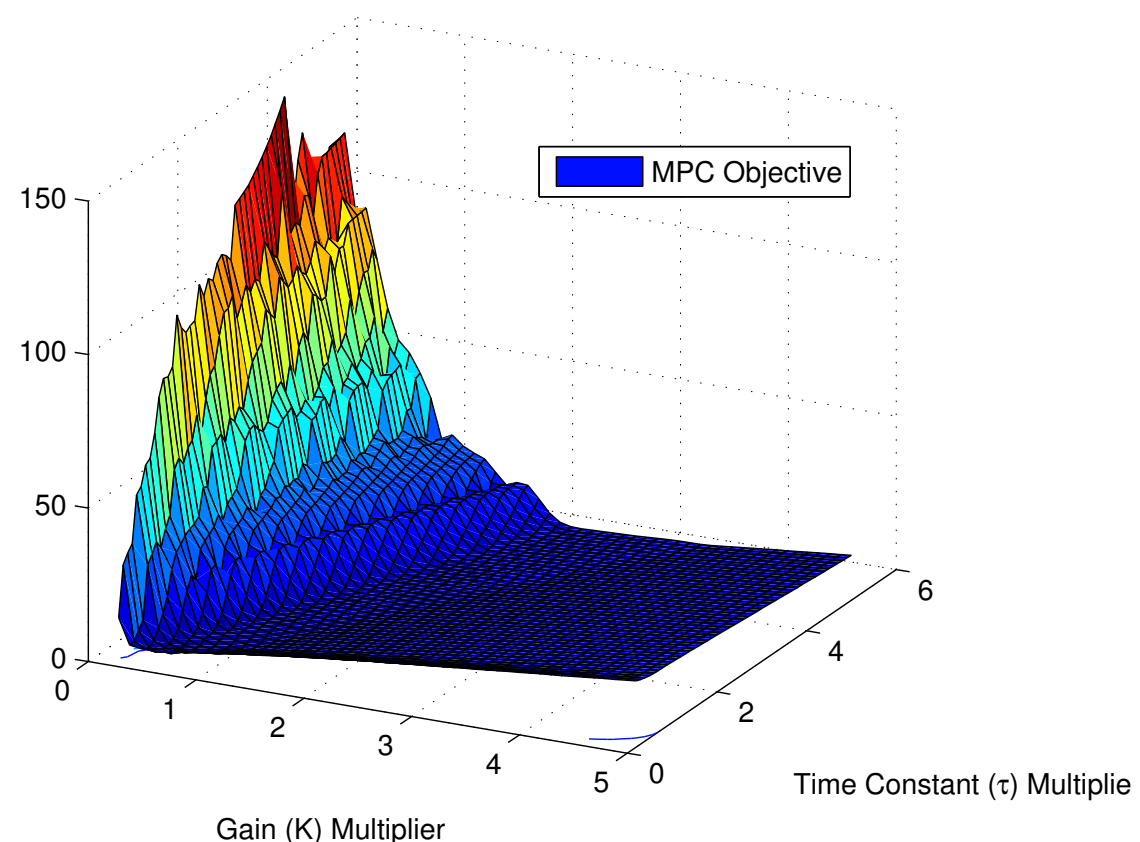

Fig. 14 Mismatch with too low model gain and too high time constant favor controller instability.

\section{Concluding Remarks}

There is a recent increase in data availability in the oil and gas industry due to advances in technology, improved networking, and regulatory requirements that require additional monitoring. When measurements are viewed individually they provide insight into the true state of the process, but do not offer a holistic view of the process. When combined with a process model, the data provides an increased understanding of unmeasured disturbances or unmeasured states. This alignment of measurements and model predictions is accomplished with a variety of techniques ranging from a simple bias update to large-scale optimization approaches. Two optimization approaches discussed in this paper include MHE with $\ell_{1}$-norm and squared errors. Efficient solution of the MHE approach is important for solving large-scale problems of industrial significance. Simultaneous solution of the objective function and model equations is an efficient approach to solving large-scale models for data reconciliation. In many cases, the objective in state and parameter estimation is to obtain a model that is sufficiently accurate for predictive control applications. Model mismatch with a gain that is too low or time constant that is too high may lead to unsatisfactory control performance. 


\section{References}

1. Abul-el-zeet, Z., Roberts, P.: Enhancing model predictive control using dynamic data reconciliation. AIChE Journal 48(2), 324-333 (2002)

2. Albuquerque, J., Biegler, L.: Decomposition algorithms for on-line estimation with nonlinear models. Computers and Chemical Engineering 19(10), 1031-1039 (1995)

3. Allgöwer, F., Badgwell, T.A., Qin, J.S., Rawlings, J.B., Wright, S.J.: Nonlinear predictive control and moving horizon estimationan introductory overview. In: Advances in control, pp. 391-449. Springer (1999)

4. Biegler, L., Yang, X., Fischer, G.: Advances in sensitivity-based nonlinear model predictive control and dynamic real-time optimization. Journal of Process Control (0), - (2015). DOI http://dx.doi.org/10.1016/j.jprocont.2015.02.001

5. Binder, T., Blank, L., Bock, H., Burlisch, R., Dahmen, W., Diehl, M., Kronseder, T., Marquardt, W. Schlöder, J., Stryk, O.: Online Optimization of Large Scale Systems, chap. Introduction to model based optimization of chemical processes on moving horizons, pp. 295-339. Springer-Verlag Berlin Heidelberg (2001)

6. Brower, D., Hedengren, J., Loegering, C., Brower, A., Witherow, K., Winter, K.: Fiber optic monitoring of subsea equipment. In: Ocean, Offshore \& Arctic Engineering OMAE, 84143. Rio de Janiero, Brazil (2012)

7. Carey, G., Finlayson, B.: Othogonal collocation on finite elements. Chemical Engineering Science 30, 587-596 (1975)

8. Darby, M., Nikolaou, M., Jones, J., Nicholson, D.: RTO: An overview and assessment of current practice. Journal of Process Control 21, 874-884 (2011)

9. Eaton, A., Safdarnejad, S., Hedengren, J., Moffat, K., Hubbell, C., Brower, D., Brower, A.: Postinstalled fiber optic pressure sensors on subsea production risers for severe slugging control. In: ASME 34th International Conference on Ocean, Offshore, and Arctic Engineering (OMAE), 42196. St. John's, Newfoundland, Canada (2015)

10. Ellis, M., Durand, H., Christofides, P.D.: A tutorial review of economic model predictive control methods. Journal of Process Control 24(8), 1156 - 1178 (2014). DOI http://dx.doi.org/10.1016/j.jprocont.2014.03.010. Economic nonlinear model predictive control

11. Findeisen, R., Allgöwer, F., Biegler, L.: Assessment and future directions of nonlinear model predictive control. Springer-Verlag, Berlin (2007)

12. Hallac, B., Kayvanloo, K., Hedengren, J., Hecker, W., Argyle, M.: An Optimized Simulation Model for Iron-Based Fischer-Tropsch Catalyst Design: Transfer Limitations as Functions of Operating and Design Conditions. Chemical Engineering Journal 263, 268-279 (2015)

13. Haseltine, E., Rawlings, J.: Critical evaluation of extended kalman filtering and moving-horizon estimation. Ind. Eng. Chem. Res. 44(8), 2451-2460 (2005)

14. Hedengren, J.: Advanced process monitoring. Control Engineering Practice p. submitted (2012)

15. Hedengren, J., Brower, D.: Advanced process monitoring of flow assurance with fiber optics. In: AIChE Spring Meeting. Houston, TX (2012)

16. Hedengren, J., Edgar, T.: Order reduction of large scale dae models. In: IFAC 16th World Congress. Prague, Czechoslovakia (2005)

17. Hedengren, J., Edgar, T.: Moving horizon estimation - the explicit solution. In: Proceedings of Chemical Process Control (CPC) VII Conference. Lake Louise, Alberta, Canada (2006)

18. Hedengren, J.D., Shishavan, R.A., Powell, K.M., Edgar, T.F.: Nonlinear modeling, estimation and predictive control in $\{$ APMonitor $\}$. Computers \& Chemical Engineering 70, 133 - 148 (2014). DOI http://dx.doi.org/10.1016/j.compchemeng.2014.04.013. Manfred Morari Special Issue

19. Hutin, R., Tennent, R., Kashikar, S.: New mud pulse telemetry techniques for deepwater applications and improved real-time data capabilities. In: SPE/IADC Drilling Conference, 67762-MS. Society of Petroleum Engineers, Amsterdam, Netherlands (2001)

20. Jacobsen, L., Spivey, B., Hedengren, J.: Model predictive control with a rigorous model of a solid oxide fuel cell. In: Proceedings of the American Control Conference (ACC), pp. 3747-3752. Washington, D.C. (2013)

21. Jang, S., Joseph, B., Mukai, H.: Comparison of two approaches to on-line parameter and state estimation of nonlinear systems. Ind. Eng. Chem. Process Des. Dev. 25, 809-814 (1986)

22. Jeffrey, K., Forward, K.: Improvements with broadband networked drill string. Digital Energy Journal 18, 7-8 (2009) 
23. Jensen, K., Hedengren, J.: Improved load following of a boiler with advanced process control. In: AIChE Spring Meeting. Houston, TX (2012)

24. Kelly, J., Hedengren, J.: A steady-state detection (SSD) algorithm to detect non-stationary drifts in processes. Journal of Process Control 23(3), 326-331 (2013)

25. Kelly, J., Zyngier, D.: Continuously improve the performance of planning and scheduling models with parameter feedback. In: FOCAPO 08 - Foundations of Computer Aided Process Operations. Boston, MA (2008)

26. Lambert, R., Nascu, I., Pistikopoulos, E.: Simultaneous reduced order multi-parametric moving horizon estimation and model predictive control. Dynamics and Control of Process Systems 10(1), 267278 (2013)

27. Liebman, M., Edgar, T., Lasdon, L.: Efficient data reconciliation and estimation for dynamic processes using nonlinear programming techniques. Computers and Chemical Engineering 16, 963-986 (1992)

28. Long, R., Veeningen, D.: Networked drill pipe offers along-string pressure evaluation in real time. World Oil pp. 91-94 (2011)

29. Moraal, P., Grizzle, J.: Observer design for nonlinear systems with discrete-time measurements. IEEE Transactions on Automatic Control 40(3), 395-404 (1995)

30. Muske, K.R., Badgwell, T.A.: Disturbance modeling for offset-free linear model predictive control. Journal of Process Control 12, 617-632 (2002)

31. Nybø, R., Frøyen, J., Lauvsnes, A.D., Korsvold, T., Choate, M.: The overlooked drilling hazard: Decision making from bad data. In: SPE Intelligent Energy International, SPE-150306. Society of Petroleum Engineers, Utrecht, The Netherlands (2012)

32. Odelson, B., Rajamani, M., Rawlings, J.: A new autocovariance least-squares method for estimating noise covariances. Automatica 42(2), 303-308 (2006)

33. Pannocchia, G., Kerrigan, E.: Offset-free control of constrained linear discrete-time systems subject to persistent unmeasured disturbances. In: Proceedings of the 42nd IEEE Conference on Decision and Control, pp. 3911-3916. Maui, Hawaii (2003)

34. Pannocchia, G., Rawlings, J.: Disturbance models for offset-free MPC control. AIChE Journal 49(2), 426-437 (2002)

35. Pixton, D.S., Craig, A.: Drillstring network 2.0: An enhanced drillstring network based on 100 wells of experience. In: IADC/SPE Drilling Conference and Exhibition, SPE-167965-MS. Society of Petroleum Engineers, Fort Worth, TX (2014). DOI http://dx.doi.org/10.2118/167965-MS

36. Pixton, D.S., Shishavan, R.A., Perez, H.D., Hedengren, J.D., Craig, A.: Addressing ubo and mpd challenges with wired drill pipe telemetry. In: SPE/IADC Managed Pressure Drilling \& Underbalanced Operations Conference \& Exhibition, SPE-168953-MS. Society of Petroleum Engineers (2014)

37. Powell, K.M., Hedengren, J.D., Edgar, T.F.: Dynamic optimization of a hybrid solar thermal and fossil fuel system. Solar Energy 108, 210 - 218 (2014). DOI http://dx.doi.org/10.1016/j.solener.2014.07.004

38. Prata, D.M., Lima, E.L., Pinto, J.C.: Nonlinear dynamic data reconciliation in real time in actual processes. In: C.A.O.d.N. Rita Maria de Brito Alves, E.C. Biscaia (eds.) 10th International Symposium on Process Systems Engineering: Part A, Computer Aided Chemical Engineering, vol. 27, pp. 47 54. Elsevier (2009). DOI http://dx.doi.org/10.1016/S1570-7946(09)70228-7

39. Qin, S., Badgwell, T.: Nonlinear Model Predictive Control, chap. An overview of nonlinear model predictive control applications, pp. 369-392. Birkhäuser Verlag, Boston, MA (2000)

40. Ramamurthi, Y., Sistu, P., Bequette, B.: Control-relevant dynamic data reconciliation and parameter estimation. Computers and Chemical Engineering 17(1), 41-59 (1993)

41. Ramlal, J., Naidoo, V., Allsford, K., Hedengren, J.: Moving horizon estimation for an industrial gas phase polymerization reactor. In: Proc. IFAC Symposium on Nonlinear Control Systems Design (NOLCOS). Pretoria, South Africa (2007)

42. Rao, C., Rawlings, J., Lee, J.: Constrained linear state estimation - a moving horizon approach. Automatica 37, 1619-1628 (2001)

43. Rawlings, J., Angeli, D., Bates, C.: Fundamentals of economic model predictive control. In: 2012 IEEE 51st Annual Conference on Decision and Control (CDC), pp. 3851-3861 (2012). DOI 10.1109/CDC.2012.6425822

44. Rawlings, J., Mayne, D.: Model predictive control: theory and design. Nob Hill Publishing, LLC, Madison, WI (2009)

45. Renfro, J., Morshedi, A., Asbjornsen, O.: Simultaneous optimization and solution of systems described by differential/algebraic equations. Computers and Chemical Engineering 11(5), 503-517 (1987) 
46. Safdarnejad, S.M., Hedengren, J.D., Baxter, L.L.: Plant-level dynamic optimization of cryogenic carbon capture with conventional and renewable power sources. Applied Energy 149(0), 354 - 366 (2015). DOI http://dx.doi.org/10.1016/j.apenergy.2015.03.100

47. Safdarnejad, S.M., Hedengren, J.D., Lewis, N.R., Haseltine, E.: Initialization strategies for optimization of dynamic systems. Computers \& Chemical Engineering (0), - (2015). DOI http://dx.doi.org/10.1016/j.compchemeng.2015.04.016

48. Shishavan, R.A., Hubbell, C., Perez, H., Hedengren, J.D., Pixton, D.S.: Combined rate of penetration and pressure regulation for drilling optimization using high speed telemetry. SPE Drilling \& Completion Journal (SPE-170275-MS) (2015). DOI 10.2118/170275-PA

49. Shishavan, R.A., Hubbell, C., Perez, H., Hedengren, J.D., Pixton, D.S., Pink, A.P.: Multivariate control for managed pressure drilling systems using high speed telemetry. In: SPE Annual Technical Conference and Exhibition, SPE-170962-MS. Society of Petroleum Engineers, Amsterdam, The Netherlands (2014). DOI http://dx.doi.org/10.2118/170962-MS

50. Soderstrom, T., Edgar, T., Russo, L., Young, R.: Industrial application of a large-scale dynamic data reconciliation strategy. Industrial and Engineering Chemistry Research 39, 1683-1693 (2000)

51. Soroush, M.: State and parameter estimations and their applications in process control. Computers and Chemical Engineering 23, 229-245 (1998)

52. Spivey, B., Hedengren, J., Edgar, T.: Constrained nonlinear estimation for industrial process fouling. Industrial \& Engineering Chemistry Research 49(17), 7824-7831 (2010)

53. Sugiura, J., Samuel, R., Oppelt, J., Ostermeyer, G.P., Hedengren, J.D., Pastusek, P.: Drilling modeling and simulation: Current state and future goals. SPE/IADC-173045-MS. London, UK (2015)

54. Sui, D., Nybø, R., Gola, G., Roverso, D., Hoffmann, M.: Ensemble methods for process monitoring in oil and gas industry operations. Journal of Natural Gas Science and Engineering 3(6), 748 - 753 (2011). DOI http://dx.doi.org/10.1016/j.jngse.2011.05.004. Artificial Intelligence and Data Mining

55. Sun, L., Hedengren, J.D., Beard, R.W.: Optimal trajectory generation using model predictive control for aerially towed cable systems. Journal of Guidance, Control, and Dynamics 37(2), 525-539 (2014)

56. Taylor, J., del Pilar Moreno, R.: Nonlinear dynamic data reconciliation: In-depth case study. In: 2013 IEEE International Conference on Control Applications (CCA), pp. 746-753 (2013). DOI 10.1109/CCA.2013.6662839

57. Vachhani, P., Rengaswamy, R., Gangwal, V., Narasimhan, S.: Recursive estimation in constrained nonlinear dynamical systems. AIChE Journal 51(3), 946-959 (2005)

58. Zavala, V., Biegler, L.: Nonlinear programming strategies for state estimation and model predictive control. In: L. Magni, D. Raimondo, F. Allgöwer (eds.) Nonlinear Model Predictive Control, Lecture Notes in Control and Information Sciences, vol. 384, pp. 419-432. Springer Berlin Heidelberg (2009)

Acknowledgements The authors would like to acknowledge the financial and technical assistance of National Oilwell Varco (NOV) and SINTEF in projects related to modeling and control design for automated drilling systems. 